\title{
Prevalencia de la migraña en estudiantes de educación básica y media de Mérida, Venezuela
}

\author{
Juana Rondón, ${ }^{1}$ Alix Padrón-Freytez, ${ }^{1}$ Remy Rada F. ${ }^{1}$
}

RESUMEN Objetivos. Determinar la prevalencia de la migraña en una población estudiantil del Municipio Libertador (Mérida, Venezuela), el efecto incapacitante de la cefalea en general y la proporción de individuos que se automedican.

Métodos. Se aplicó una autoencuesta a una muestra de 1714 alumnos de 10 a 21 años de edad seleccionada por muestreo estratificado y aleatorio simple.

Resultados. La prevalencia de la cefalea fue de $84,4 \%$, y la de la migraña de 16,8\%. La migraña no tuvo un efecto incapacitante importante en esta población. De los estudiantes con cefalea, $69,2 \%$ no solicitaron asistencia médica y 80,3\% se automedicaron.

Conclusiones. La migraña es muy frecuente y las cifras de automedicación y carencia de asistencia médica indican que debe existir un déficit de cobertura de los servicios de salud y desinformación de la población que ocasiona un subregistro de la cefalea.

Palabras clave Cefalalgia, migraña, adolescencia, prevalencia, automedicación.

En la práctica neurológica diaria la cefalea es el primer motivo de consulta, pero los datos epidemiológicos que se conocen proceden mayormente de series clínicas (1), lo cual sugiere que debe haber un subregistro importante porque buena parte de la población no solicita ayuda médica y recurre a la au-

\footnotetext{
1 Clinica de Cefaleas. Unidad de Neurología, Instituto Autónomo Hospital Universitario de Los Andes (IAHULA), Av 16 de Septiembre. Mérida, Venezuela. Toda la correspondencia debe ser enviada a Juana Rondón, a la siguiente dirección: Unidad de Neurología, Instituto Autónomo Hospital Universitario de Los Andes (IAHULA), Av 16 de Septiembre, Mérida, Venezuela. FAX 58-74403045. Correo electrónico: zucam@cantv.net
}

tomedicación (1-4). Por este motivo, nos propusimos realizar un estudio poblacional para determinar la prevalencia de la cefalea, y específicamente de la migraña, en adolescentes (5) estudiantes de educación básica y media de 10-21 años de edad. Como objetivos adicionales, también analizamos la relación de la migraña con variables demográficas, la asociación entre la migraña y la incapacidad, evaluada a través del absentismo escolar, y la frecuencia de la intervención médica y de la automedicación en el grupo estudiado. Algunas investigaciones poblacionales realizadas en los últimos años han situado la prevalencia de la migraña entre 6,6 y 18,5\% $(1,4,6-9)$.

\section{MÉTODOS}

El estudio se realizó en el Municipio Autónomo Libertador del Estado de Mérida, que cuenta con 178290 habitantes (XII Censo de Población y Vivienda) y cuya población estudiantil de educación básica y media para el año escolar 1990-1991 era de 14277 alumnos. La muestra, de 1714 estudiantes de 10 a 21 años de edad, se seleccionó mediante muestreo estratificado en una primera etapa, correspondiente a la selección de las instituciones educativas por parroquias del municipio, y muestreo aleatorio simple en una segunda etapa (10), para seleccionar el aula por año escolar. 
Los estudiantes seleccionados respondieron a una encuesta en la que registraron sus datos de identificación, variables demográficas (edad, sexo, procedencia, estado civil, tenencia de la vivienda, nivel educativo de la madre), las características de la cefalea (edad de aparición, localización, características del dolor, frecuencia, etc.), la incapacidad, medida a través del absentismo escolar, y la asistencia médica y automedicación. La aplicabilidad y factibilidad de esta autoencuesta fueron probadas previamente mediante un estudio piloto realizado en institutos educativos del Municipio Autónomo Campo Elías, situado a 20 $\mathrm{km}$ del casco urbano de Mérida. La validez del instrumento de trabajo se estableció con una sensibilidad de 85\% y una especificidad de $90 \%$ en pacientes de la Clínica de Cefaleas del Instituto Autónomo Hospital Universitario de Los Andes (IAHULA) $(10,11)$. La encuesta fue aplicada durante los meses de mayo y junio de 1991 (fecha próxima a concluir el año escolar 1990-1991).

La definición operacional de migraña sin aura consistió en cefalea unilateral, pulsátil, acompañada de náuseas o vómitos, o fonofobia o fotofobia, asociada con situaciones precipitantes que se tomaron en consideración si los síntomas concomitantes no eran concluyentes (12-16). En la migraña con aura la cefalea debía estar precedida de los síntomas neurológicos focales que caracterizan el aura. En vista de que se trataba de un estudio poblacional, los antecedentes familiares de mi-

CUADRO 1. Prevalencia de la migraña según la edad, sexo y tipo de migraña en 1714 alumnos de educación básica y media. Municipio Autónomo Libertador. Mérida, Venezuela, 1991

\begin{tabular}{|c|c|c|c|c|c|c|}
\hline & \multicolumn{2}{|c|}{ Migraña con aura } & \multicolumn{2}{|c|}{ Migraña sin aura } & \multicolumn{2}{|c|}{ Total } \\
\hline & No. & $\%$ & No. & $\%$ & No. & $\%$ \\
\hline Total & 87 & 5,1 & 201 & 11,7 & 288 & 16,8 \\
\hline \multicolumn{7}{|l|}{ Edad (en años) } \\
\hline $10-12$ & 0 & 0 & 3 & 0,2 & 3 & 0,2 \\
\hline $13-15$ & 43 & 2,5 & 97 & 5,7 & 140 & 8,2 \\
\hline $16-18$ & 40 & 2,3 & 91 & 5,3 & 131 & 7,6 \\
\hline $19-21$ & 4 & 0,2 & 10 & 0,6 & 14 & 0,8 \\
\hline \multicolumn{7}{|l|}{ Sexo } \\
\hline Masculino $(n=718)$ & 15 & 0,9 & 52 & 3,0 & 67 & 9,3 \\
\hline Femenino $(n=996)$ & 72 & 4,2 & 149 & 8,7 & 221 & 22,2 \\
\hline
\end{tabular}

graña se incluyeron como criterio que apoyaba el diagnóstico.

\section{RESULTADOS}

La muestra estudiada quedó integrada por 718 individuos del sexo masculino $(41,9 \%)$ y $996 \mathrm{del}$ sexo femenino (58,1\%), 1607 de los cuales $(93,8 \%)$ tenían edades comprendidas entre los 13 y los 18 años. Se registraron antecedentes de cefalea en $84,4 \%$ de la población estudiada, con claro predominio del sexo femenino $(61,7 \%)$.

La prevalencia de la migraña fue de $22,2 \%$ en el sexo femenino y $9,33 \%$ en el masculino. La migraña sin aura fue más frecuente que la migraña con aura y el grupo de edad más afectado por ambos tipos de migraña fue el de 13 a 18 años, que era el más numeroso (cuadro 1).
En cuanto a la edad de inicio, la mayor frecuencia correspondió a las edades de 8 a 13 años $(76,2 \%$ ) para ambos tipos de migraña. Esta cifra fue obtenida de las respuestas de 248 estudiantes con migraña, ya que el resto no recordaba la edad de inicio.

En relación con las características del dolor, el tipo más frecuente fue la cefalea pulsátil, y en relación con la localización, el dolor unilateral alternante, seguido de la cefalea global (cuadro 2). De los 288 estudiantes con migraña, solo 260 respondieron a las preguntas sobre las características del dolor. En cuanto a la frecuencia de la migraña, predominaron los grupos con 1 ó 2 episodios/mes y 1 ó 2 episodios/semana (cuadro 3).

La migraña no fue incapacitante en poco más de la mitad de los pacientes con aura y en cerca de tres cuartos de los pacientes sin aura. La incapacidad

CUADRO 2. Distribución de la población con migraña según las características y la localización del dolor. Municipio Autónomo Libertador. Mérida, Venezuela, 1991

\begin{tabular}{|c|c|c|c|c|c|c|c|c|c|c|}
\hline \multirow[b]{3}{*}{ Característica } & \multicolumn{10}{|c|}{ Localización } \\
\hline & \multicolumn{2}{|c|}{$\begin{array}{l}\text { Unilateral } \\
\text { alternante }\end{array}$} & \multicolumn{2}{|c|}{$\begin{array}{c}\text { Unilateral } \\
\text { persistente }\end{array}$} & \multicolumn{2}{|c|}{ Orbitario } & \multicolumn{2}{|c|}{ Global } & \multicolumn{2}{|c|}{ Total } \\
\hline & No. & $\%$ & No. & $\%$ & No. & $\%$ & No. & $\%$ & No. & $\%$ \\
\hline Pulsátil & 57 & 21,9 & 10 & 3,9 & 30 & 11,5 & 30 & 11,5 & 127 & 48,9 \\
\hline Opresivo & 14 & 5,4 & 3 & 1,2 & 8 & 3,1 & 11 & 4,2 & 36 & 13,9 \\
\hline Punzante & 34 & 13,1 & 9 & 3,5 & 17 & 6,5 & 19 & 7,3 & 79 & 30,4 \\
\hline Otros & 6 & 2,3 & 1 & 0,4 & 5 & 1,9 & 6 & 2,3 & 18 & 6,9 \\
\hline Total & 111 & 42,7 & 23 & 8,8 & 60 & 23,1 & 66 & 25,4 & 260 & 100 \\
\hline
\end{tabular}


CUADRO 3. Distribución de la población con migraña según la frecuencia del dolor. Municipio Autónomo Libertador. Mérida, Venezuela, 1991

\begin{tabular}{lrr}
\hline Frecuencia & No. & $\%$ \\
\hline 1-2/semana & 130 & 44,2 \\
1-2/mes & 13 & 44,6 \\
2-4/año & 27 & 9,2 \\
1/año & 6 & 2,0 \\
Total & 294 & 100 \\
\hline
\end{tabular}

moderada a grave fue más frecuente en los casos con aura. Más de la mitad de los pacientes de ambos grupos no buscaron asistencia médica. La mayoría de los individuos (cerca de dos tercios con aura y tres cuartos sin aura) se automedicaron (cuadro 4).

\section{DISCUSIÓN}

La encuesta realizada reveló que $84,4 \%$ de la población sufre de cefalea, con clara preponderancia del sexo femenino $(61,7 \%)$ con respecto al masculino $(38,3 \%)$. Nuestra investigación no puede compararse de forma estricta con las de Linet (4) y Forgays (6), ya que difiere en criterios operacionales, pero en todas se persigue la determinación de la frecuencia o prevalencia de la cefalea y de la migraña, por lo que pueden establecerse comparaciones en algunas variables. La prevalencia puntual en nuestro estudio fue inferior a la registrada por Linet et al. (4) (93,4\%), quienes también determinaron la prevalencia de período, que fue menor $(62,7 \%)$ y comparable a la de otras investigaciones (17-19). No determinamos la prevalencia de período debido a las características psicológicas propias de la edad de nuestra población (poca importancia otorgada a las enfermedades, que hace que no recuerden bien la fecha de aparición del trastorno, falta de autonomía para acudir al médico o recibir medicación e incluso para decidir no asistir a clases) $(5,19)$ y al hecho de que se desconoce el intervalo de tiempo ideal para determinar la prevalencia de la migraña, ya que los intervalos utilizados hasta ahora han suscitado dudas $(1,2,4,19,20)$.

La prevalencia de la migraña $(16,8 \%)$ fue superior a la citada en la mayoría de los estudios poblacionales $(1,4,7-9,21)$. Sin embargo, hay que señalar que aún no se ha establecido definitivamente el patrón de la migraña en escolares y adolescentes, lo cual dificulta el diagnóstico clínico y, más aún, su identificación epidemiológica $(3,19,22-24)$. Con respecto al sexo se observó un predominio femenino a razón de 3,3:1, hecho que se ha observado también en la mayoría de las poblaciones estudiadas $(4,7,9)$.

La prevalencia de la migraña con aura $(5,1 \%)$ y de la migraña sin aura $(11,7 \%)$ resultan igualmente elevadas en comparación con otros estudios publicados $(1,4,17)$, y el grupo de edad más afectado fue el de 13 a 18 años (94,1\%). Las manifestaciones comenzaron más frecuentemente $(76,2 \%)$ entre los 8 y los 13 años de edad, resultado similar al obtenido por otros autores, aunque difiere ligeramente del estudio prospectivo de Bille (25), en el que la edad de inicio más frecuente fue a los 5 años.

Clásicamente se ha descrito la migraña como una cefalea hemicraneana, pulsátil (3, 9, 14-16, 26-29), y los criterios de clasificación vigentes (13) así lo mantienen. Aunque estamos de acuerdo en que no se ha establecido el patrón típico de la migraña a estas edades, en nuestra investigación el dolor pulsátil (48,9\%) de localización unilateral alternante $(42,7 \%)$ fue el más frecuente, en concordancia con lo observado por Linet (4).

A diferencia de otras investigaciones, la gravedad del dolor que caracteriza a la migraña $(2,9,18,20,26,27)$ y produce incapacidad fue sustancial-

CUADRO 4. Distribución de la población con cefalea según el grado de incapacidad, la asistencia médica, la automedicación y el tipo de cefalea. Municipio Autónomo Libertador. Mérida, Venezuela, 1991

\begin{tabular}{|c|c|c|c|c|c|c|c|c|}
\hline & \multicolumn{2}{|c|}{ Migraña con aura } & \multicolumn{2}{|c|}{ Migraña sin aura } & \multicolumn{2}{|c|}{ Otras cefaleas } & \multicolumn{2}{|c|}{ Total } \\
\hline & No. & $\%$ & No. & $\%$ & No. & $\%$ & No. & $\%$ \\
\hline \multicolumn{9}{|l|}{ Incapacidad ${ }^{a}$} \\
\hline Leve & 11 & 12,2 & 27 & 13,4 & 119 & 10,4 & 157 & 10,9 \\
\hline Moderada & 14 & 15,6 & 15 & 7,5 & 109 & 9,5 & 138 & 9,6 \\
\hline Grave & 16 & 17,8 & 16 & 8,0 & 145 & 12,6 & 177 & 12,3 \\
\hline No & 51 & 58,6 & 139 & 69,9 & 796 & 69,9 & 986 & 69,2 \\
\hline Sí & 36 & 41,4 & 60 & 30,2 & 343 & 30,1 & 439 & 30,8 \\
\hline Total & 87 & 100 & 199 & 100 & 1139 & 100 & 1425 & 100 \\
\hline \multicolumn{9}{|c|}{ Automedicación } \\
\hline No & 31 & 33,0 & 53 & 26,2 & 30 & 5,7 & 114 & 13,8 \\
\hline Sí & 63 & 67,0 & 149 & 73,8 & 499 & 94,3 & 711 & 86,2 \\
\hline Total & 94 & 100 & 202 & 100 & 529 & 100 & 1286 & 100 \\
\hline
\end{tabular}

a La incapacidad se evaluó por el absentismo escolar: grave (1 día), moderado (medio día) o leve (2 horas). 
mente baja en nuestra investigación $(17,8 \%$ en la migraña con aura y $7 \%$ en la migraña sin aura). Estos resultados contradicen la "definición conductual" de migraña, aunque podrían atribuirse a las características propias del adolescente, que le impiden precipitarse en el ciclo dolorangustia del adulto $(24,27,29,30)$.

Finalmente, en la población con cefalea existe una tendencia a automedicarse y a prescindir de la asistencia médica, lo cual ocasiona un subregistro de la enfermedad $(1,14,31)$. Nuestros resultados refuerzan la afirmación anterior, ya que $69,2 \%$ de la población con cefalea nunca había recibido asistencia médica, cifra que supera a las descritas en estudios similares. Se ob- servó además una elevada proporción de automedicación $(80,2 \%)$, probablemente debido a la cobertura deficiente y desorganización de los servicios de salud, aunadas a la falta de información de la población y a la venta de medicamentos sin prescripción médica, que conduce no pocas veces a la dependencia e intoxicación medicamentosa $(14,31)$.

En conclusión, los hallazgos de este estudio poblacional sugieren que la cefalea constituye un problema de salud pública por su elevada frecuencia $\mathrm{y}$ que compromete a una población muy joven, en edad productiva, que no es atendida adecuadamente por deficiencia de los servicios de salud, y que esto ocasiona subregistro y automedi- cación, con consecuencias probablemente graves. La elevada prevalencia de la migraña en esta población destaca su importancia, aunque hay que tener en cuenta que los criterios de clasificación aplicados en estudios de población como este probablemente no sean tan precisos como los que podrían aplicarse en una consulta de neurología, reafirmando las dificultades para establecer diagnósticos exactos en estudios poblacionales grandes. Para refinar y comprender más claramente la evolución natural de la migraña en estas poblaciones será necesario realizar estudios analíticos prospectivos concurrentes y una evaluación posterior de los datos obtenidos.

\section{REFERENCIAS}

1. Linet MS, Stewart WF. Migraine headache: epidemiologic perspectives. Epidemiol Rev 1984;6:107-139.

2. Ziegler DK. Headache: public health problem. Neurol Clin 1990;8:781-791.

3. Diamond, S. Migraine headaches. Med Clin North Am 1991;75:545-566.

4. Linet MS, Stewart WF, Celentano DD, Ziegler D, Sprecher M. An epidemiologic study of headache among adolescents and young adults. JAMA 1989;261:2211-2216.

5. Brown E, Hendee WR. Adolescent health. Synopsis of a conference. Am J Dis Child 1989; 193:466.

6. Forgays DG, Rzewnicki R, Ober AJ, Forgays DK. Headache in college students: a comparison of four populations. Headache 1993;33: 182-190.

7. Nikiforow R. Features of migrainecomparison of a questionnaire study and a neurologist-examined random sample. Cephalalgia 1981;1:157-166.

8. Nikiforow R. Headache in a random sample of 200 persons: a clinical study of a population in northern Finland. Cephalalgia 1981;1: 99-107.

9. D'Alessandro R, Benassi G, Lenzi PL, Gamberini G, Sacquegna T, De Carolis P, et al. Epidemiology of headache in the Republic of San Marino. J Neurol Neurosurg Psychiatry 1988;51:21-27.

10. Lilienfield AM, Lilienfield D. Fundamentos de epidemiología. 2a. ed. Nueva York:Fondo Educativo Interamericano; 1980.

11. Mausner JS, Bahn AK. Epidemiología (2. ${ }^{a}$ reimpresión). México: Editorial Interamericana; 1980.
12. Campbell JK. Manifestations of migraine. Neurol Clin 1990;8:841-855.

13. Headache Classification Committee of the International Headache Society. Classification and diagnostic criteria for headache disorders, cranial neuralgias and facial pain. Cephalalgia 1988;8(Supl 7);1-96.

14. Diamond S, Freitag FG, Solomon GD, Millstein E. Migraine headache. Working for the best outcome. Postgrad Med 1987;81:174-183.

15. Sacks O. Understanding a common disorder. Expanded and updated. First Paperback Printing California. University of California Press. 1986; p. 270.

16. Graham JR. Migraine clinical aspects. En Handbook of clinical neurology. Vol. 5: Headaches and cranial neuralgias. $1 .^{a}$ ed. Amsterdam: North-Holland Publishing Company; 1978. pp. 45-58.

17. Goldstein M, Chen TC. The epidemiology of disabling headache. Adv Neurol 1982;33: 377-390.

18. Duckro PN, Tait RC, Margolis RB. Prevalence of very severe headache in a large US metropolitan area. Cephalalgia 1989;9:199-205.

19. Rothner AD. Headaches in adolescents. Diagnosis and management. Med Clin North Am 1991;75:653-660.

20. Cheng XM, Ziegler DK, Li SC, Dai QS, Chandra V, Schoenberg BS. A prevalence survey of 'incapacitating headache' in the People's Republic of China. Neurology 1986;36:831-834.

21. Ponce DP. Estudios de prevalencia de transtornos neurológicos en Lezama. Estudios Neuroepidemiológicos en Venezuela. Caracas: Departamento de Enfermedades Neuro- lógicas, Ministerio de Sanidad y Asistencia Social; 1985. pp. 16-38.

22. Elser JM, Woody RC. Migraine headache in the infant and young child. Headache 1990;30:366-368.

23. Saper JR. Migraine. I. Classification and pathogenesis. JAMA 1978;239:2380-2383.

24. Mackenzie RG. Approach to the adolescent in the clinical setting. Med Clin North Am 1990;74:1085-1095.

25. Bille B. Migraine in schoolchildren. Acta Paediatr Scand 1962;51(Supl. 136):T151.

26. Merikangas KR, Angst J, Isler $H$. Migraine and psychopathology. Results of the Zurich cohort study of young adults. Arch Gen Psychiatry 1990;47:849-853.

27. Andrasik F. Psychologic and behavioral aspects of chronic headache. Neurol Clin 1990;8: 961-976.

28. Rothner AD. Headaches in children and adolescents. Classification and recommendations. Postgrad Med 1987;81:223-230.

29. Blau JN, Dexter SL. The site of pain origin during migraine attacks. Cephalalgia 1981; 1:143-147.

30. Smith MS. Psychosomatic symptoms in adolescence. Med Clin North Am 1990;74: 1121-1134.

31. Granella F, Farina S, Malferrari G, Manzoni GC. Drug abuse in chronic headache: a clinicoepidemiologic study. Cephalalgia 1987;7: 15-19.

Manuscrito recibido el 15 de junio de 2000. Aceptado para publicación, tras revisión, el 15 de diciembre de 2000. 
ABSTRACT Objective. The epidemiological impact of headaches in populations is not adequately known since most of the data come from clinical studies. Therefore, we decided to survey a population of students in the municipality of Libertador, in the state of

The prevalence of migraines among primary- and secondary-school students in Mérida, Venezuela
Mérida, Venezuela, to determine the prevalence of migraines, the incapacitating effect of headaches in general, and the proportion of individuals who self-medicate.

Methods. We first used stratified sampling to choose schools in the municipality, and then simple random sampling to select which classrooms to survey. A total of 1714 students, ranging in age from 10 to 21 years old, completed a survey.

Results. Among the students, $84.4 \%$ of them reported having had headaches, and $16.8 \%$ of them reported experiencing migraines. We did not find a noticeable incapacitating effect of migraines in this population. Of the students who had had headaches, $69.2 \%$ of them did not request medical care, and $80.3 \%$ of them reported selfmedicating.

Conclusions. From these results we conclude that migraines are very frequent among this population. Judging from the levels of self-medication and the lack of medical attention for headaches, we believe there is a shortfall in health services coverage and a deficiency in public information, which together lead to an underreporting of headaches.

Internados en Investigación en el Harvard Center for Society and Health

Fecha: Septiembre de 2001

Lugar: Boston, Massachusetts, Estados Unidos de América

La Escuela de Salud Pública de la Universidad de Harvard en Estados Unidos, en colaboración con la OPS/OMS, ofrece internados a dos investigadores latinoamericanos o caribeños para estudiar, en su Harvard Center for Society and Health, el tema de la inequidad en materia de salud. Esta iniciativa ha sido suscitada por la evidente escasez de estudios y la necesidad de fortalecer la investigación en este campo en América Latina y el Caribe. Cada internado, que tendrá una duración de nueve meses (un año académico) a partir de septiembre de 2001, se acompaña de un subsidio mensual de US\$2 000.00, seguro de salud y pasaje aéreo de ida y vuelta. Puede aspirar cualquier investigador latinoamericano o caribeño que conozca el inglés y que esté afiliado con una institución académica o centro de investigación en su país, que posea un título universitario o esté cursando estudios de doctorado, y que tenga un mínimo de cinco años de experiencia en el campo de la investigación. La fecha límite para el recibo de las propuestas, que deben enviarse por correo electrónico, es el 15 de mayo de 2001. Las propuestas deben tener no más de 15 páginas redactadas en inglés y un resumen de un máximo de 250 palabras.

Información

PAHOHarvard Research Internships

Correo electrónico: rgp@paho.org 